Preface

\title{
Zika Virus: A Race to Prevent a Reproductive Crisis
}

Jeanne Sheffield, $M D^{1}$ James Segars, $M D^{2}$

${ }^{1}$ Division of Maternal-Fetal Medicine, Department of Gynecology and Obstetrics, Johns Hopkins University School of Medicine,

Baltimore, Maryland

2 Division of Reproductive Sciences and Women's Health Research, Department of Gynecology and Obstetrics, Johns Hopkins University School of Medicine, Baltimore, Maryland

Semin Reprod Med 2016;34:259-260

Mankind is in the midst of a reproductive epidemic quite unlike any that has come before. In short, the world's population is nonimmune for the Zika virus, and it is clear that not only is it likely the virus will afflict a large percentage of the population, but also the virus affects reproduction of our species. Simply put, infection during pregnancy is associated with severe and lasting fetal effects, including microcephaly and cerebral defects leading to life-long consequences for the affected offspring. What makes Zika different from prior epidemics we have faced is that the population is nonimmune and the virus is transmitted by three vectors: females of Aedes aegypti, females of Aedes albopictus and both females and males of Homo sapiens. Thus, spread of Zika cannot be interrupted by eradication of all disease vectors.

Fortunately, prior epidemics caused by viruses transmitted by A. aegypti, such as yellow fever, and sexual transmission of human immunodeficiency virus provide a rough blueprint of some effective strategies. We know a great deal about $A$. aegypti from the pioneering work of the Cuban doctor, Carlos Finlay, who first recognized the mosquito as a vector; the Johns Hopkins Researcher, Jesse Lazear, who died proving Finlay's hypothesis; and Army doctors, Walter Reed and William Gorgas. ${ }^{1-3}$ This species resides in close contact with humans, breeds in still water found around residences, bites throughout the day, does not transit large distances, and reproduction of the female mosquito requires a blood meal. Although Aedes is endemic throughout many regions of the world, including South and Central America, Florida, and the Southern United States, simple measures implemented by Dr. Gorgas over a century ago can eliminate spread of Zika virus by the mosquito. Communication and education are important tools at our disposal. Regrettably, human sexual transmission of Zika cannot be stopped by these simple and time-proven measures.
Because of the severe reproductive consequences of infection in pregnancy, we are engaged in a race to develop effective diagnostic tests, immunization strategies, public health and education programs to thwart the spread of disease, and prevent infection during pregnancy. Prevention is the key. Unfortunately, we lack detailed knowledge and fundamental understanding of this unusual epidemic.

Both the guest editors face the consequences of Zika infection daily in our subspecialties of maternal-fetal medicine and reproductive endocrinology and infertility. We recognize that this issue of Seminars in Reproductive Medicine will

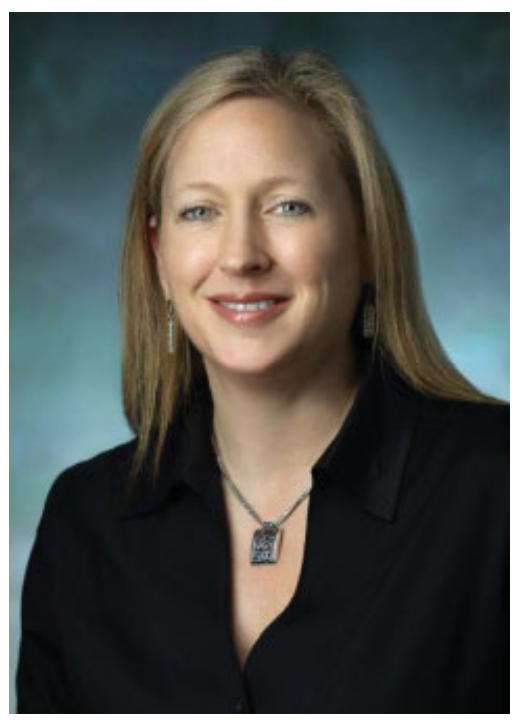

Jeanne Sheffield, MD

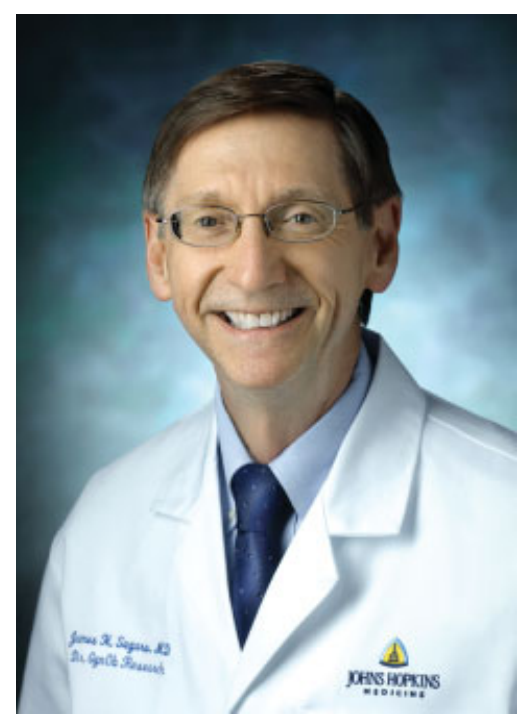

James Segars, MD

story, and as such, it will depict a work in progress. It will report a milestone and not the final story. Yet, we believe it is important to educate providers, frame the progress that has been made, and identify crucial and pressing research needs. Most importantly, we emphasize the critical need for research

Address for correspondence James Segars, MD, Division of Reproductive Sciences and Women's Health Research, Department of Gynecology and Obstetrics, Johns Hopkins University School of Medicine, 624 Ross, 720 Rutland, Baltimore, MD 21205

(e-mail: jsegars2@jhmi.edu).
Issue Theme The Zika Virus Pandemic and Reproductive Medicine; Guest Editors, Jeanne Sheffield, MD and James Segars, MD
DOI http://dx.doi.org/ 10.1055/s-0036-1592065. ISSN 1526-8004.
Copyright $₫ 2016$ by Thieme Medical Publishers, Inc., 333 Seventh Avenue, New York, NY 10001, USA. Tel: +1(212) 584-4662. 
and programmatic funding to address the crisis. If nothing is done, thousands of children and families will face life-long impairment because of Zika with consequences as significant as polio.

We have organized the issue to provide the reader with an up-to-date synopsis of the Zika epidemic by leaders in the field. In the first article, Dr. Kawwass will review the epidemiology of Zika and focus on the public health and history of this unusual arborvirus. The placenta and fetus are clearly a target and Dr: Burd will provide an up-to-date understanding of the molecular mechanisms of Zika viral infection. There are significant neurologic consequences associated with infection and experts Drs. Laura Sofia Muños and Carlos Pardo discuss the pathogenesis of Guillain-Barré syndrome, encephalitis, and myelitis. The reproductive and prenatal effects of Zika and management of the pregnant woman are crucial to understand and the article by Drs. Elandou and Sheffield provides current understanding and guidelines. Sexual transmission of Zika has implications for couples seeking to build a family, including couples with infertility and the risks and current guidelines are reviewed in the fifth article in this issue.

Prevention of disease is the key and the last three articles examine strategies to prevent or mitigate Zika infection. Because of the possibility for gamete infection, and the risks of contamination in the assisted reproductive technology laboratory, Drs. Davis, Ball, Bracero, and Washington review current guidelines and practices to mitigate the threat. The current status of vaccine development and the possible timeline and strategies are discussed by Dr. Durbin. Finally, and arguably most important, Dr. Burke addresses family planning and strategies that can be immediately implemented to thwart the Zika epidemic.

Despite the progress that has been made in a comparatively brief period of time, there are many unknowns regarding Zika and the current epidemic. It is our hope that by raising awareness and education that progress toward the goal of eradication of the virus or prevention of disease can be realized. We envision a future wherein an effective vaccine will be developed, the nonimmune state can be readily diagnosed, and the reproductive threat of Zika will be greatly reduced or eliminated. However, this will take time, and until then, education and prevention are the only medical strategies to fight the virus.

\section{References}

1 Finlay C. The mosquito hypothetically considered as an agent in the transmission of yellow fever poison. Yale J Biol Med 1937;9(6): 589-604

2 Chaves-Carballo E. Carlos Finlay and yellow fever: triumph over adversity. Mil Med 2005;170(10):881-885

3 Pierce JR, Writer JV. Yellow Jack: How Yellow Fever Ravaged America and Walter Reed Discovered Its Deadly Secrets. Hoboken, NJ: John Wiley \& Sons, Inc; 2005 